\title{
Analysis of Temperature Field in the Flow Field and the Stress Field of the Friction Plate of the Wet Clutch
}

\author{
Jingang Liu ${ }^{1}$, Ming $\mathrm{Mu}^{1, *}$, Jianwen Chen ${ }^{2}$ and Quan $\mathrm{Li}^{2}$ \\ ${ }^{1}$ School of mechanical engineering, Xiangtan University, Xiangtan 411105, China \\ ${ }^{2}$ Hunan Jianglurongda Vehicle Transmission Co. Ltd, Changsha Hunan 410100, China \\ ${ }^{*}$ Corresponding Author: Ming Mu
}

Keywords: Wet Clutch; The Simulation Calculation. The Flow Field. The Stress Field; Temperature Field; Thermal-Mechanical Coupling.

\begin{abstract}
Aiming at the friction temperature is too high, excessive thermal stress problems of wet clutch engagement process, establish the three-dimensional model of wet multi disc clutch, using ANSYS workbench finite element software, the calculation process considering various displacement constraints, the boundary conditions are established. Convection field, stress field without oil groove and different types of the friction plate, and the temperature field and stress field of the friction plate are simulated. The research results provide a reference for the optimization design of wet multi disc clutch and the establishment of the clutch thermal management system.
\end{abstract}

\section{Introduction}

Wet multi disc clutch work is through the active friction plate and the driven friction plate with each other, achieve the same speed in order to achieve the purpose of shift gears. In the course of the work, the friction between the active friction plate and the driven friction plate is produced by the friction heat, and the heat stress is produced due to the uneven heat dissipation problem. The temperature and thermal stress of wet multi disc clutch are greatly influenced by the positive pressure distribution of special friction surface. When the pressure distribution is not uniform, the distribution of the heat produced by the friction surface is not uniform. This can lead to high local temperature and thermal stress. When the temperature is too high, it is easy to produce thermos elastic instability, which can lead to the phenomenon of warping and bonding, and even the sintering and shedding of the friction material.

So analyze the wet multi-disc clutch friction plate has the vital significance. The difficulty for the analysis of the friction plate is clutch during work, stress not only from friction and friction will produce thermal stress. At the same time, friction heat and cooling liquid will be taken away, this time may also need to consider the motion of the fluid coolant, the transmission torque and the dynamic interaction of many complex factors such as the liquid viscous transmission and thermal elastic problem of the results.

\section{Flow Field}

\subsection{Fluid control equation}

Fluid flow to be controlled by the law of conservation of physical. The basic conservation laws include mass conservation law, momentum conservation law. Assumes that the lubricating oil is incompressible fluid, in Cartesian coordinates, adiabatic turbulent fluid control equation can be expressed as the mass conservation equation, momentum conservation equation (1) (2).

$$
\begin{gathered}
\nabla \cdot u=0 \\
\frac{\partial u_{i}}{\partial t}+\nabla \cdot\left(u_{i} u\right)=-\frac{1}{\rho} \frac{\partial p}{\partial x_{i}}+\mu \nabla \cdot \operatorname{grad}\left(u_{i}\right)+S_{i}
\end{gathered}
$$

In the formula:

u-velocity vector, 
$\nabla \cdot u=\partial u / \partial x+\partial v / \partial y+\partial w / \partial z$

$u_{i}$-speed $\mathrm{x}$ along the axis $\mathrm{u}, \mathrm{y}, \mathrm{z}$ component $\mathrm{u}, \mathrm{V}, \mathrm{W}$, which I take 1, 2, 3;

$x_{i}$-the coordinate axes $\mathrm{x}, \mathrm{y}, \mathrm{z}$ in the Cartesian coordinate system, where i takes 1, 2, 3;

$\rho$-lubricating oil density;

$\mu$-dynamic viscosity;

p-lubricating oil pressure;

Si-The components of SuSvSw the generalized source term along the axes $\mathrm{x}, \mathrm{y}, \mathrm{z}$.

\subsection{Calculation parameters}

Density of lubricating oil in clutch is $880 \mathrm{~kg} / \mathrm{m}^{3}$, Specific Heat is $1600 \mathrm{~J} \cdot\left(\mathrm{kg} \cdot{ }^{\circ} \mathrm{C}\right)^{-1}$, Thermal Conductivity is $0.144 \mathrm{~W} \cdot\left(\mathrm{m} \cdot{ }^{\circ} \mathrm{C}\right)^{-1}$.

Table 1. Interface parameters of common wet clutch radial oil groove

\begin{tabular}{|c|c|c|c|}
\hline \multicolumn{4}{|c|}{ Cross - sectional Parameters of Three Kinds of Common Wet Clutches } \\
\hline type of oil groove & Section angle/ $\theta$ & Groove width/mm & Number of oil sumps \\
\hline Triangular oil groove & 90 & 1 & 38 \\
\hline Trapezoidal oil groove & 120 & 1 & 48 \\
\hline Arc oil groove & 120 & 1.75 & 48 \\
\hline
\end{tabular}

\subsection{Analysis of calculation results}

Analysis of flow field distribution in wet clutch engagement process using ANSYS WORKBENCH and FLUID SOFTWARE. Semi-implicit algorithm (SIMPLE) for steady-state solution of the turbulence model, the oil tank alone out to simplify the overall calculation. Setting up import and export and the flow field grid in the tank, the pressure field and velocity field are solved by means of adjusting the relaxation factor in order to accelerate the convergence of the model and to analyze the flow field. Figure1, Figure2, Figure3, Figure4, Figure5, Figure 6 shows.

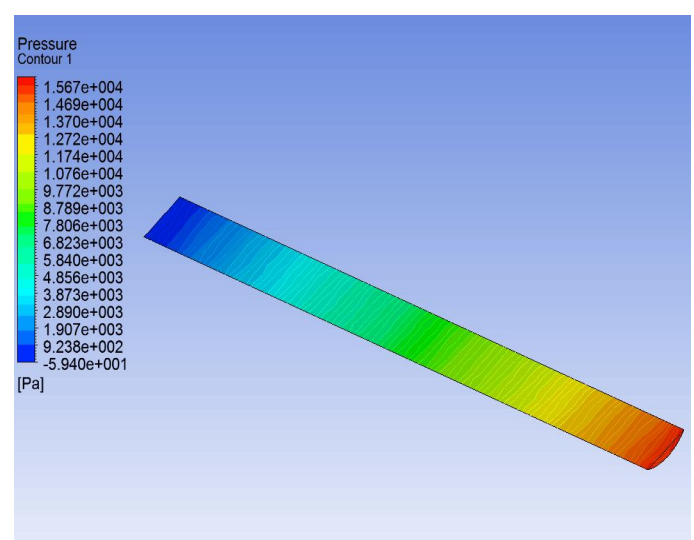

Figure 1. Arc groove pressure field 


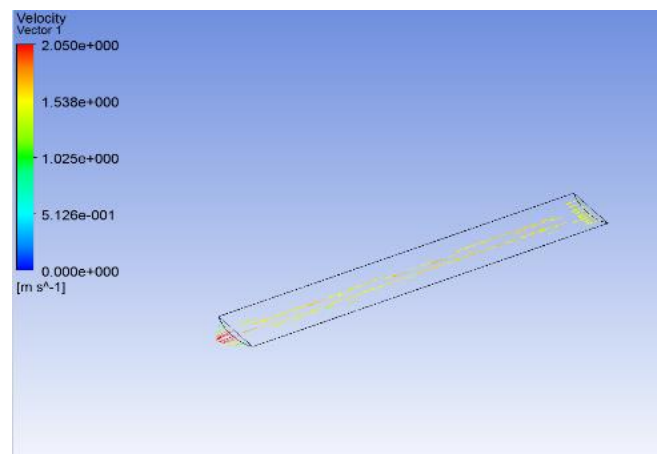

Figure 2. Arc groove velocity field

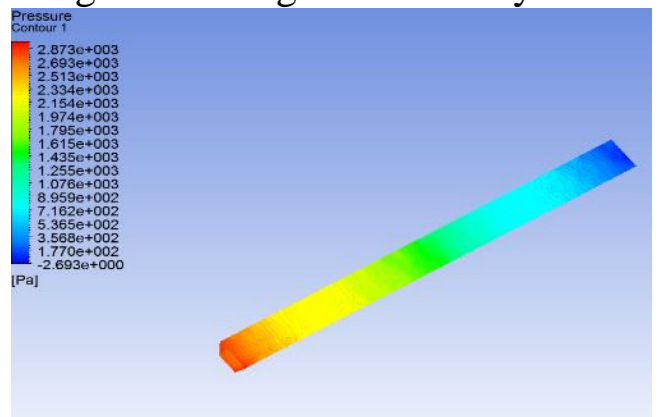

Figure 3. Trapezoidal groove pressure field

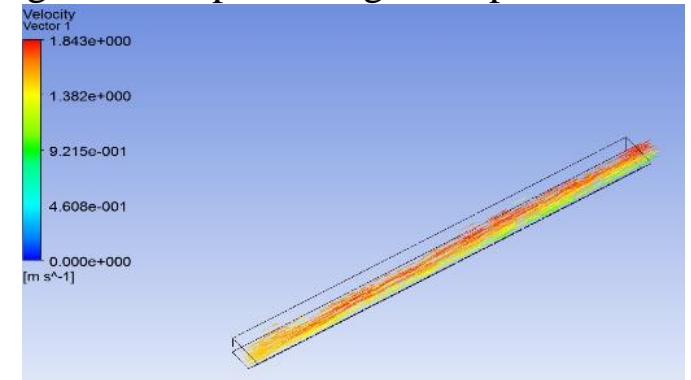

Figure 4. Trapezoidal groove velocity field

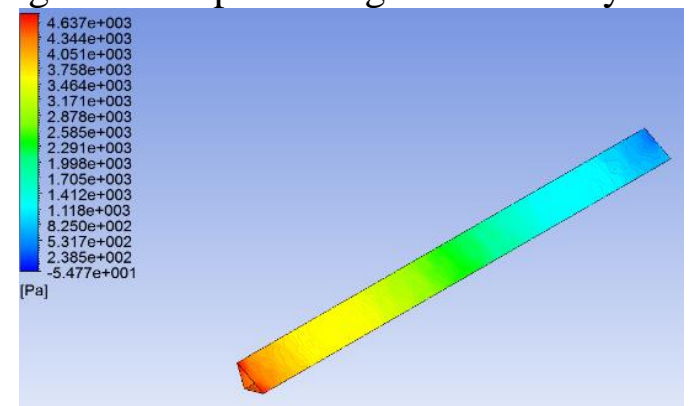

Figure 5. Triangular groove pressure field

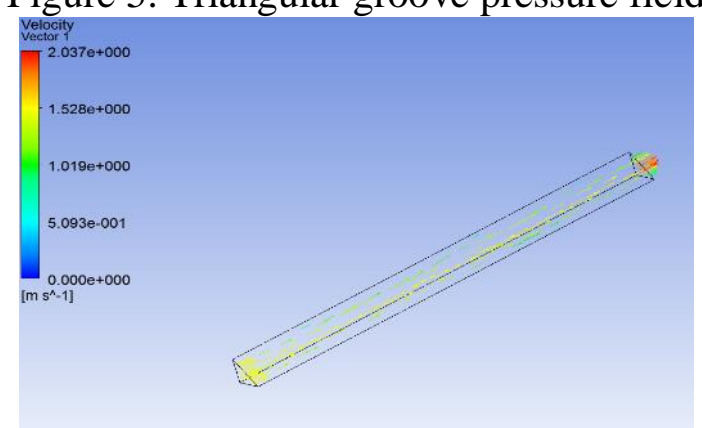

Figure 6. Triangular groove velocity field

Figure 1, Figure 3 and Figure 5 for the three oil road lubricating oil pressure cloud Figure. As can be seen, the pressure of three oil groove at the inlet pressure is large, the pressure loss is large at the exit. The maximum pressure of the arc groove is $1.18 \mathrm{e}+002 \mathrm{pa}$, the maximum pressure of the 
trapezoidal groove is $2.873 \mathrm{e}+003 \mathrm{pa}$, and the maximum pressure of the triangle is $4.637 \mathrm{e}+003 \mathrm{pa}$. Comparison of the three kinds of groove, the pressure of the outlet of the arc groove is the most. Figure 2, Figure 4, Figure 6 for the lube of three kinds of oil road velocity vector Figure. From the Figure, the maximum velocity of emulsified oil in the three kinds of oil circuit structure are all at the exit point. The maximum velocity of the arc groove is $2.05 \mathrm{~m} / \mathrm{s}$, the maximum speed of the trapezoidal groove is $1.843 \mathrm{~m} / \mathrm{s}$, the maximum speed of the triangular groove is $2.037 \mathrm{~m} / \mathrm{s}$. In general, the greater the inlet pressure, the greater the oil speed.

\section{The stress field}

\subsection{Analysis and calculation of stress field of friction pair}

Mechanical elastic strain $\varepsilon$ formed by steel disc under the action of mechanical force. According to the linear theory of stress, the stress and strain caused by mechanical forces can be superimposed with the stress and strain induced by temperature, the total strain between the two interactions is:

$$
\varepsilon=\varepsilon_{j}+\varepsilon_{T}
$$

The stress vector and strain have the following relationship:

$$
\sigma=D_{\varepsilon}
$$

In the formula, $\mathrm{D}$ is the material elastic matrix.

The type (1) into (2) type can gain the friction element stress of the steel:

$$
\sigma=D\left(\varepsilon_{j}+\varepsilon_{T}\right)
$$

The equilibrium relationship between the contact pressure $\mathrm{p}(\mathrm{x}, \mathrm{y}, \mathrm{z})$ and the element stress $\sigma$ on the joints is:

$$
\int_{v} B \sigma d V=p(x, y \cdot z)
$$

In the formula, the $\mathrm{B}$ is the transformation matrix between the element strain and the nodal displacement, and the $\mathrm{V}$ is the volume of the steel disc.

The stress field distribution of friction plate and steel sheet can be calculated after the mechanical stress and thermal elastic stress overlap each other in the process of sliding by the formula (3) (6). The contact between the friction plate and the steel sheet is elastic contact, cannot use the iterative algorithm to check the contact between the two, In case of contact, the contact should be immediately generated constraint and the force on the node.

\subsection{Calculation parameters}

Friction material is paper based friction material. In the analysis of stress field, friction factor is 0.3 , constant speed is $2500 \mathrm{rad} / \mathrm{min}$, and constant pressure is $2.1 \mathrm{MPa}$. Mesh generation of the model by using tetrahedral element. Material parameters of friction sheet, structure size are distributed in table 2 and table 3.

Table 2. Size parameter of friction plate structure

\begin{tabular}{|c|c|c|}
\hline \multicolumn{2}{|c|}{ Simplified structure dimension parameters of friction plate } \\
\hline \multirow{3}{*}{ The friction plate } & Name & Size \\
\cline { 2 - 3 } & Inner diameter of friction plate & $104 \mathrm{~mm}$ \\
\cline { 2 - 3 } & Outer diameter of friction plate & $130 \mathrm{~mm}$ \\
\hline \multirow{3}{*}{ Steel disc } & Friction core thickness & $1.8 \mathrm{~mm}$ \\
\cline { 2 - 3 } & Inside diameter of steel disc & $104 \mathrm{~mm}$ \\
\cline { 2 - 3 } & Outer diameter of steel disc & $130 \mathrm{~mm}$ \\
\hline
\end{tabular}


Table 3. Material parameters of friction sheet

\begin{tabular}{|c|c|c|}
\hline Characteristic parameter & Friction material & $\begin{array}{c}\text { Dual steel sheet and friction } \\
\text { core board }\end{array}$ \\
\hline Density $/ \rho / m g \cdot m^{-3}$ & 833 & 7850 \\
\hline Elastic modulus/GPa & 0.588 & 206 \\
\hline Poisson ratio & 0.12 & 0.3 \\
\hline Thermal conductivity $/ w \cdot(m \cdot C)^{-1}$ & 0.24 & 52 \\
\hline Specific heat $/{ }^{J} \cdot\left(\mathrm{kg} \cdot{ }^{\cdot} \mathrm{C}\right)^{-1}$ & 1740 & 490 \\
\hline Coefficient of expansion $/{ }^{10^{-6} \cdot\left({ }^{\circ} \mathrm{C}\right)^{-1}}$ & 1.7 & 10.6 \\
\hline
\end{tabular}

\subsection{Analysis of calculation results}

Analysis of stress field distribution in wet clutch engagement process by using workbench ANSYS software. A fixed constraint is imposed on the friction core face, stress is applied to the steel disc; steel disc set rotation angle velocity, it analog relative rotation; the same boundary condition is applied to the contact surface of the master-driven friction disc. The results of friction pairs after calculated can be gain on Figure 7, Figure 8, Figure 9, Figure 10, Figure 11, Figure 12, Figure 13, Figure 14, Figure 15, and Figure 16.

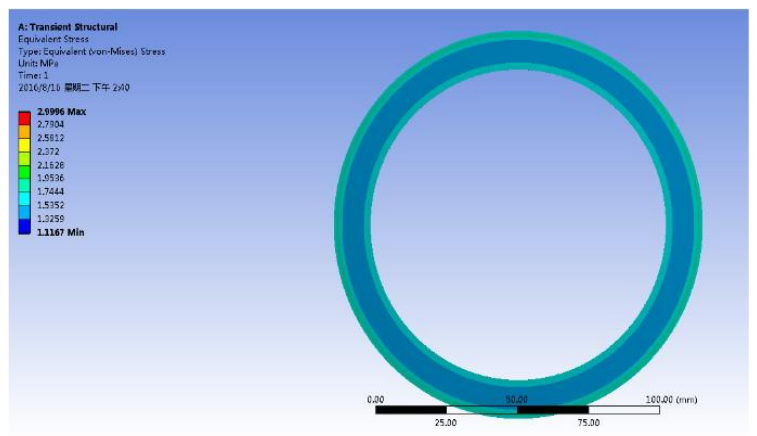

Figure 7. Stress distribution of the contact surface of steel disc without oil

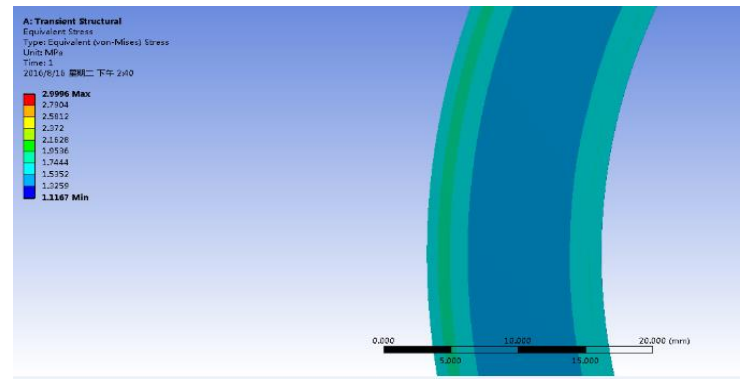

Figure 8. Stress distribution of the Partial contact surface of steel disc without oil

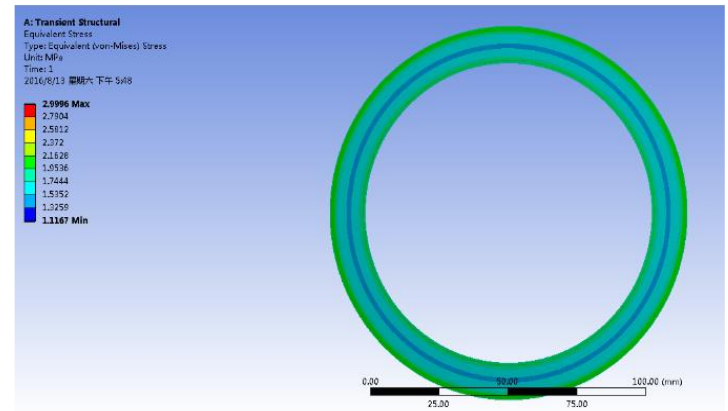

Figure 9. Stress distribution of contact surface of friction plate without oil groove 


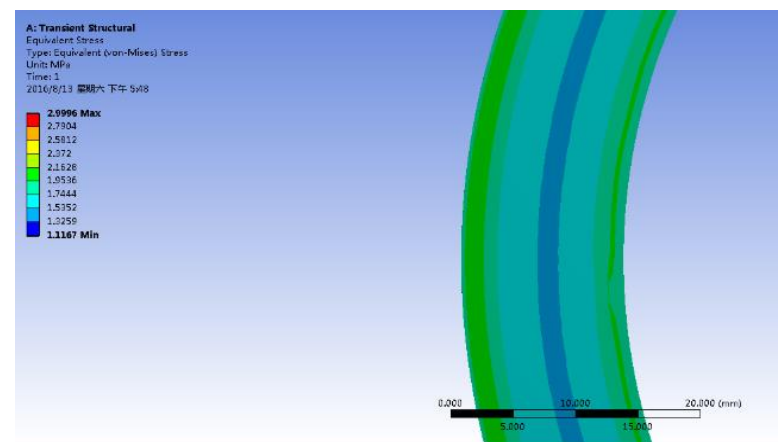

Figure 10. Stress distribution of partial contact surface of friction plate without oil groove

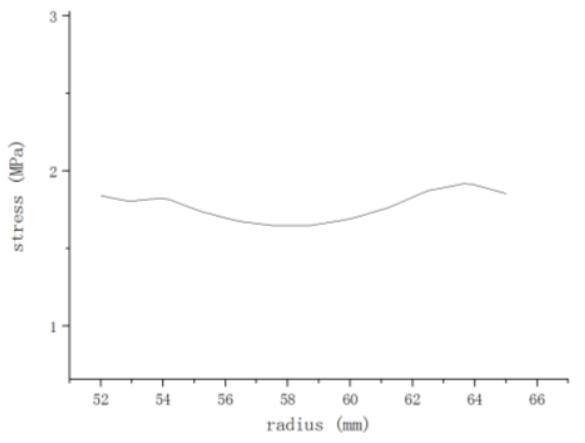

Figure 11. The stress distribution curve of the inner diameter to the outer diameter of the contact surface of the friction plate

From the Figure, the effect of stress on the steel sheet is larger when the oil free tank, the stress distribution of steel sheet is more complicated, but the inner stress of the inner ring is maximized. The stress distribution of the friction plate is stable in the middle part, the inner and outer rings increase gradually.

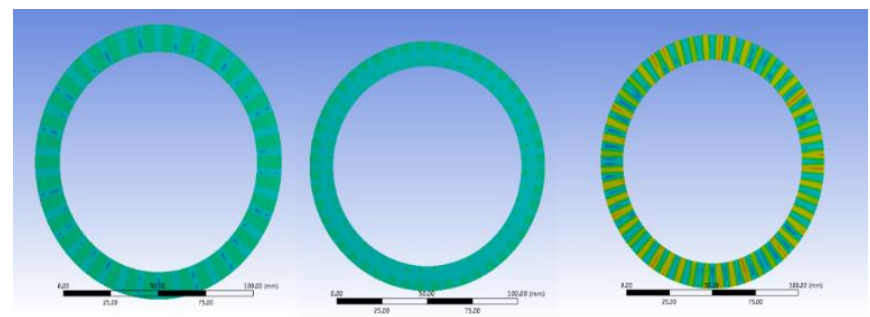

Triangular oil groove Trapezoidal oil groove Arc oil groove

Figure 12. Stress distribution of contact surface of three kinds of steel sheets

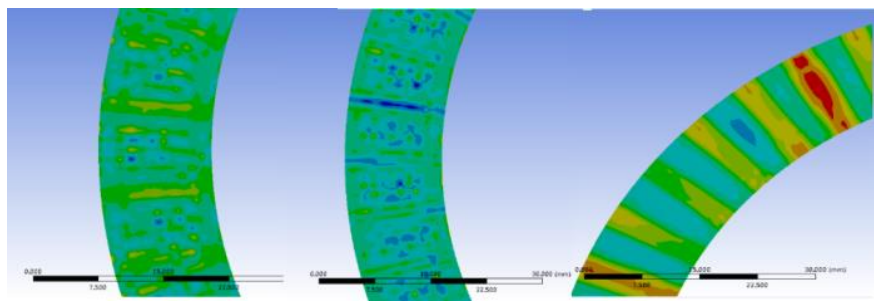

Triangular oil groove Trapezoidal oil groove Arc oil groove

Figure 13. Stress distribution of partial contact surface of three kinds of steel sheets

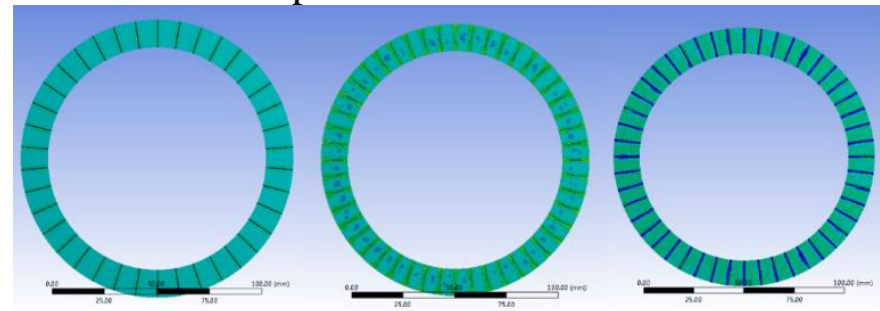

Triangular oil groove Trapezoidal oil groove Arc oil groove

Figure 14. Stress distribution of contact surface of three kinds of friction plates 


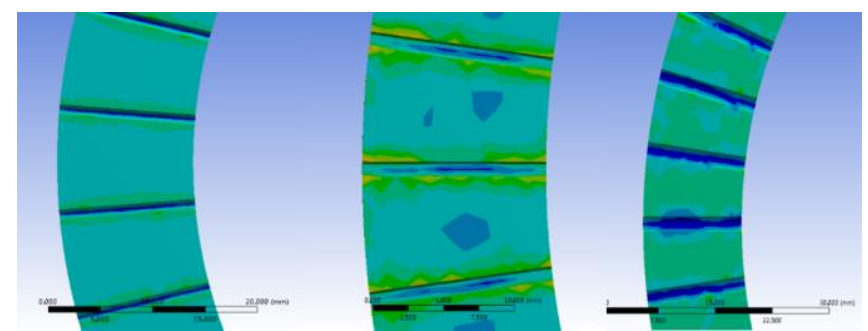

Triangular oil groove Trapezoidal oil groove Arc oil groove

Figure 15. Stress distribution of partial contact surface of three kinds of friction plate

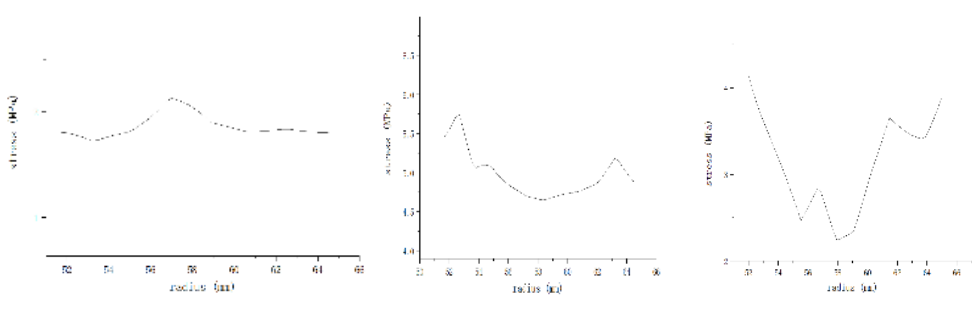

Triangular oil groove Trapezoidal oil groove Arc oil groove

Figure 16. The stress distribution curve of the inner diameter to outer diameter of the contact surface of the three kinds of friction plate

As shown in Figure 15, in the distribution of stress field on the friction plate, the stress distribution of the triangular oil groove and the trapezoidal groove is high on both sides, lower in the tank connected, while the arc-shaped oil groove is opposite. Due to the high local stress, the friction and wear of wet clutch friction disk can be produced. Figure 16 shows the stress distribution from the inner diameter to the outer diameter at the edge of each friction plate sump, the state can be summarized as lower on middle and higher on both sides.

\section{Stress field and temperature field under coupled condition}

\subsection{Analysis and Calculation of Friction Heat}

It is assumed that the mass wear of the steel discs and the friction plate is neglected during the thermal analysis, according to the first law of thermodynamics:

$$
Q-W=\Delta K+\Delta U+\Delta P
$$

In the formula:

Q - Heat;

W- Work;

$\Delta K$ - System kinetic energy;

$\Delta U$ - System internal energy;

$\Delta P$ - System potential energy.

\subsection{Calculate the parameters}

In the stress field and the temperature field under the coupling action, the friction factor is 0.15 , consider the rotational speed difference as shown in Figure 17, and apply pressure to the steel sheet as shown in Figure 18.

There are only one pair of friction pairs in this paper, while in the actual situation, there are many pairs of friction, the grooves are also modeled separately in the flow field, this simplifies the analysis and facilitates calculation, making the results more intuitive.

\subsection{Analysis of calculation results}

Analyze temperature field distribution during wet clutch engagement through ANSYS workbench. The inner and outer torus and friction contact surface were applied to the corresponding convective heat transfer, simulation of friction cooling, applying a fixed constraint on the friction core face, a piston thrust is applied to the steel sheet. A control point is set in the center of the steel sheet. A rotational displacement is applied, analog relative rotation, to achieve the heat source, convection, constraints, and mechanical external coupling load, the temperature field and the stress field are 
solved under the condition of the thermos - mechanical coupling. The results are shown in Figure 19, Figure 20, Figure 21, and Figure 22.

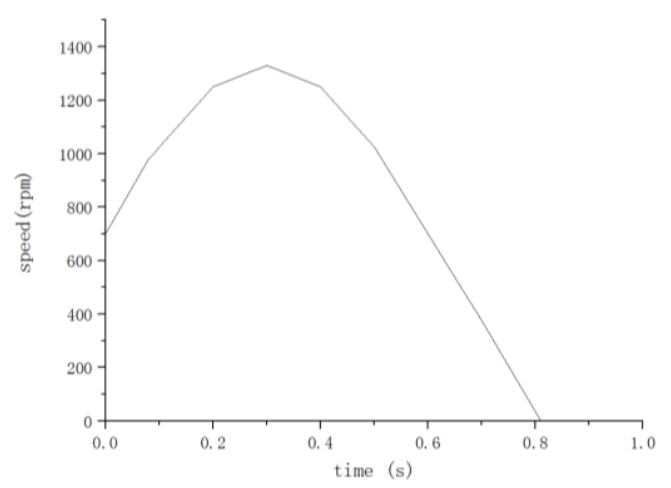

Figure 17. The applied rotational speed

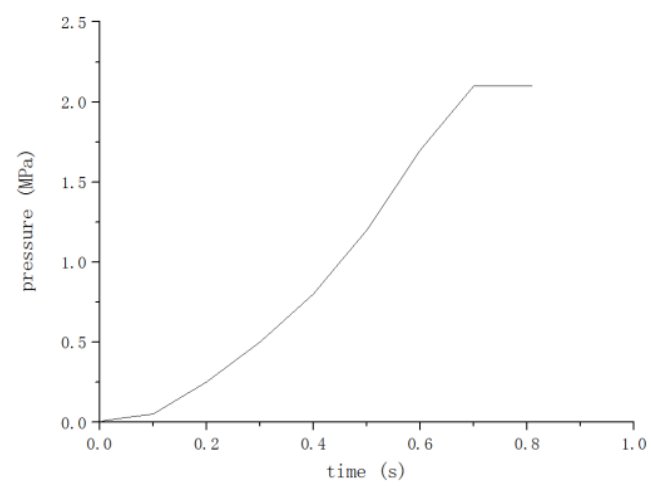

Figure 18. The applied pressure

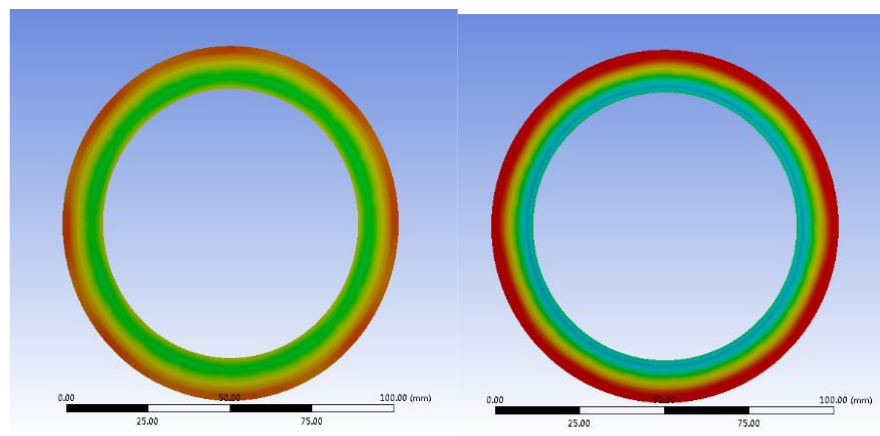

Figure 19. Stress distribution curve of front and contact surface of steel plate

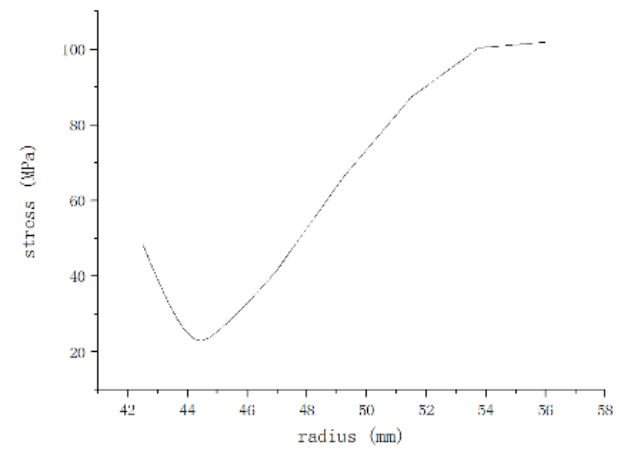

Figure 20. Stress distribution curve of steel plate contact surface 

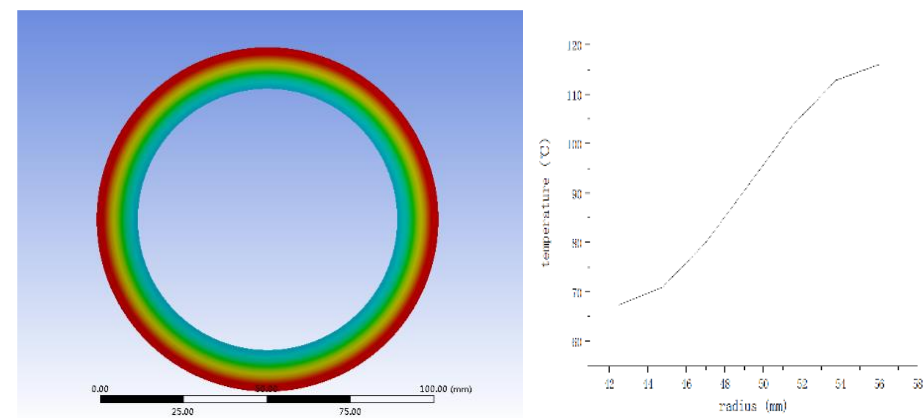

Figure 21. Distribution and curve of temperature field of steel plate contact surface
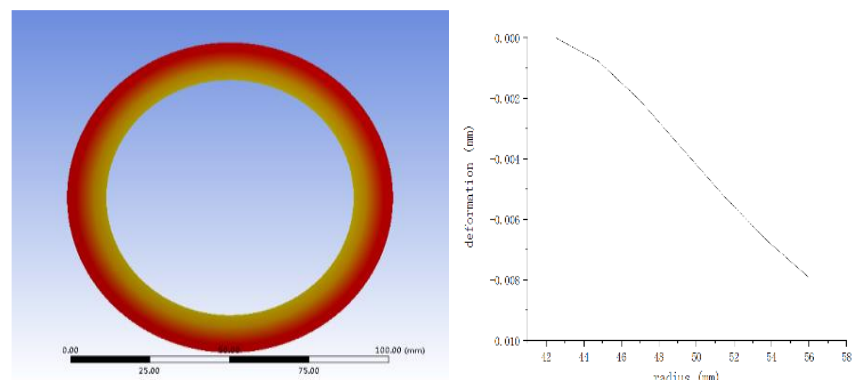

Figure 22. Full deformation distribution and curve of steel plate contact surface

As shown in Figure 19, 20, In the case of the same structure parameters of the friction pair, it can be seen from the radial distribution of stress that along the radial direction, the stress gradually increases and the stress gradient increases, the distribution is not uniform. As shown in Figure 21, the temperature profile of the dual steel sheet in the radial direction, it can be seen that along the radial direction, the heat flow input increases, the temperature increases, and the temperature gradient increases. In the deformation distribution and its curve Figure 22 can be seen, in the radial direction, the deformation in the z-axis direction is gradually increased. In summary, the edge of the friction plate is more likely to produce warped.

\section{Conclusion}

According to the above calculation results, we can see: (1) In the flow field, the effect of pressure and flow rate is that the greater the inlet pressure, the faster the flow rate.(2) According to the stress field, the stress distribution is related to the shape of the oil groove, and the stress distribution of different shapes is different, In the stress distribution of three kinds of different radial slots, The trapezoid groove shows the least stress, So trapezoidal oil tank is the best.(3) In the stress field and the temperature field under the coupling condition, under the action of stress and heat, the edge portion of the friction plate is more likely to warp.

In the actual driving process of the automobile, the adverse effects that result from the accumulation temperature of clutch heat and the increasing stress should be taken into account, consider taking additional lubrication and other management measures to protect the clutch.

\section{Acknowledgements}

This project is supported by National Natural Science Foundation of China (NO. 51475402) and Key Program of Science Research of Hunan Provincial Department of Education (NO. 15A185).

\section{References}

[1] Chen Y F et al. 2010. Research on Heat Dissipation of the Multi-disk Wet Clutch. Journal of Wuyi University. 
[2] Ding J L. 2011. Study on Thermal Field Analysis and Temperature Prediction Model of Wet Multi - plate Clutch. Chongqing University.

[3] He Z Y et al. 2010. Coupled Thermal Structure Analysis of Wet Multi - plate Friction Clutch. Mechanical research and Application.

[4] Miao Let al. 2015. Analysis on Fluid Characteristics of Friction Plate in Wet Multi-plate Clutch Based on Computational Fluid Dynamics. Atlantis-press.com.

[5] Yang Y L et al. 2014 .Analysis of Temperature Field and Stress Field of Wet Multi - plate Clutch. China Mechanical Engineering.

[6] Yang Y Q et al. 2016. Thermo - mechanical Coupling Analysis of Wet Friction Clutch Disc. Mechanical Transmission.

[7] Zhang K. 2013. Research on Thermo - mechanical Coupling Analysis and Temperature Prediction Model of Wet Multi - plate Clutch. Chongqing University.

[8] Zheng S Q et al. 2013. Improvement and finite element analysis of friction plate for 